\title{
Derivation of relativistic hydrodynamic equations consistent with relativistic Boltzmann equation by renormalization-group method
}

\author{
Kyosuke Tsumura $^{1}$ and Teiji Kunihiro ${ }^{2, a}$ \\ 1 Analysis Technology Center, Fujifilm Corporation, Kanagawa 250-0193, Japan \\ 2 Department of Physics, Kyoto University, Kyoto 606-8502, Japan
}

Received: 2 June 2012 / Revised: 27 August 2012

Published online: 27 November 2012

(C) The Author(s) 2012. This article is published with open access at Springerlink.com Communicated by T. Bíró

\begin{abstract}
We review our work on the application of the renormalization-group method to obtain first- and second-order relativistic hydrodynamics from the relativistic Boltzmann equation (RBE) as a dynamical system, with some corrections and new unpublished results. For the first-order equation, we explicitly obtain the distribution function in the asymptotic regime as the invariant manifold of the dynamical system, which turns out to be nothing but the matching condition defining the energy frame, i.e., the Landau-Lifshitz one. It is argued that the frame on which the flow of the relativistic hydrodynamic equation is defined must be the energy frame, if the dynamics should be consistent with the underlying RBE. A sketch is also given for derivation of the second-order hydrodynamic equation, i.e., extended thermodynamics, which is accomplished by extending the invariant manifold so that it is spanned by excited modes as well as the zero modes (hydrodynamic modes) of the linearized collision operator. On the basis of thus constructed resummed distribution function, we propose a novel ansatz for the functional form to be used in Grad moment method; it is shown that our theory gives the same expressions for the transport coefficients as those given in the Chapman-Enskog theory as well as the novel expressions for the relaxation times and lengths allowing natural interpretation.
\end{abstract}

\section{Introduction}

The dynamical evolution of the hot and/or dense QCD matter produced in the Relativistic Heavy Ion Collider (RHIC) at the Brookhaven National Laboratory can be well described by relativistic hydrodynamic simulations $[1$, 2 ]. It seems to be the case also for the created matter in the Large Hadron Collider (LHC) at the European Organization for Nuclear Research (CERN); see, for example, [3,4]. The suggestion that the created matter at RHIC may have only a tiny viscosity prompted an interest in the origin of the viscosity in the created matter to be described using the relativistic quantum field theory and also the dissipative hydrodynamic equations. We note that since the created matter expands, the proper dynamics for the description may change from hydrodynamics to kinetic one and vice versa $[4-9]$. The hydrodynamics is also relevant to the soft-mode dynamics [10-12] around the possible critical point(s) in QCD phase diagram [13,14]; see [15] for the latest update.

However, the theory of relativistic hydrodynamics for viscous fluids is still under debate. In fact, we can indi-

\footnotetext{
a e-mail: kunihiro@ruby.scphys.kyoto-u.ac.jp
}

cate the following problems: 1) There are ambiguities in the definition of the flow velocity [16-19]; 2) in the Eckart (particle) frame, there arises an unphysical instability of the equilibrium state [20]; 3) the so-called first-order equations lack in causality, i.e., some components of the hydrodynamic equations are of parabolic nature [21-24].

Taking the relativistic Boltzmann equation (RBE) [21, $22]$ as a typical kinetic equation, we have been exploring the basic problems with the relativistic hydrodynamics [25-27]. We note that such an approach is important also for a systematic analysis of RHIC/LHC data, because the proper dynamics for the description may change from hydrodynamics to kinetic one and vice versa, as mentioned above.

It is conjectured $[28,29]$ that the non-equilibrium process evolves through some stages of hierarchical dynamics: In the beginning of the time evolution of an isolated prepared state, the whole dynamical evolution of the system will be governed by Hamiltonian dynamics that is timereversal invariant. As the system gets old, the dynamics is relaxed into the kinetic regime where the time-evolution system is well described by kinetic equations which describe a coarse-grained slower dynamics: The Boltzmann equation for the one-body distribution function is one of 
them $[28,29]$. Usually the original time-reversal invariance is lost in the description by the kinetic equation through the coarse graining. As the system is further relaxed, the time evolution will be described in terms of the hydrodynamic quantities, i.e., the flow velocity, particle-number density, and local temperature. In this sense, the hydrodynamics is the far-infrared asymptotic dynamics of the kinetic equation.

Thus, for obtaining the proper relativistic hydrodynamic equation, it is a legitimate and natural way to start with the RBE which is Lorentz invariant and expected to be free from the causality problem [21,22]; moreover, apparent instability is not known for numerical simulations of the RBE, as far as we are aware of, and the stability is proved at least for the linearized version of it [30,31]. For analyzing problems 1) and 2) first, we derive the hydrodynamic equation $[25,27]$ from the RBE. We note that the problem is a typical reduction problem of a dynamical system in the far-infrared long-wave length limit. So, we need a powerful reduction theory for our purpose, and we shall take the renormalization-group (RG) method [3237] as such a powerful one. The reduction of dynamics can be viewed as a construction of an invariant/attractive manifold [38,39], and it has been shown [36,37] that the RG method can be nicely formulated as an elementary method for constructing the invariant manifold of a given dynamical system.

In this article, we also report on our attempt [40] to examine the causality problem (3) by deriving the so called extended thermodynamics [41-47]: Namely, we derive mesoscopic dynamics of the $\mathrm{RBE}$ by constructing the invariant/attractive manifold incorporating some fast modes as well as the zero modes of the linearized collision operator. It turns out that our theory leads to the same expressions for the transport coefficients as given by the Chapman-Enskog method [48] and also novel formulas of the relaxation times in terms of relaxation functions, which allow a natural physical interpretation of the relaxation times. Moreover, the distribution function which is explicitly constructed in our theory provides a new ansatz for the functional form of the distribution function in the Grad theory [49].

\section{Introduction to the renormalization-group method by an example}

Our approach is heavily based on the reduction theory of dynamics called the renormalization-group (RG) method [32-37], and the reliability of our theory is assured by that of the method. It is nice $[36,37]$ that the RG method can be formulated as an elementary way of construction of the invariant/attractive manifold of dynamical systems; it not only leads to asymptotic dynamics of a given equation but also extracts explicitly the differential equations governing the would-be constants appearing in the solution to the differential equation. In this section, we make an account of the RG method using a simple non-linear equation.
Let us take the Van der Pol equation as an example,

$$
\ddot{x}+x=\epsilon\left(1-x^{2}\right) \dot{x},
$$

with $\epsilon$ being small.

Let $\tilde{x}\left(t ; t_{0}\right)$ be a local solution around $t \sim \forall t_{0}$, and represent it as a perturbation series,

$$
\tilde{x}\left(t ; t_{0}\right)=\tilde{x}_{0}\left(t ; t_{0}\right)+\epsilon \tilde{x}_{1}\left(t ; t_{0}\right)+\epsilon^{2} \tilde{x}_{2}\left(t ; t_{0}\right)+\ldots
$$

In the RG method, the initial value $W\left(t_{0}\right)$ is to constitute the desired (approximate) solution in a global domain and make the invariant manifold of the system. We suppose that an exact solution is given by $x(t)$ and the initial value of $\tilde{x}\left(t ; t_{0}\right)$ at $t=t_{0}$ is set up to be $x\left(t_{0}\right)$, i.e.,

$$
W\left(t_{0}\right) \equiv \tilde{x}\left(t_{0} ; t_{0}\right)=x\left(t_{0}\right)
$$

The initial value as the exact solution should also be expanded as

$$
W\left(t_{0}\right)=W_{0}\left(t_{0}\right)+\epsilon W_{1}\left(t_{0}\right)+\epsilon^{2} W_{2}\left(t_{0}\right)+\ldots
$$

The zeroth-order equation reads

$$
\mathcal{L} \tilde{x}_{0}=0,
$$

with $\mathcal{L} \equiv \frac{\mathrm{d}^{2}}{\mathrm{~d} t^{2}}+1$. The solution may be expressed as

$$
\tilde{x}_{0}\left(t ; t_{0}\right)=A\left(t_{0}\right) \cos \left(t+\theta\left(t_{0}\right)\right),
$$

with the initial value $W\left(t_{0}\right)=\tilde{x}_{0}\left(t_{0} ; t_{0}\right)=A\left(t_{0}\right) \cos \left(t_{0}+\right.$ $\left.\theta\left(t_{0}\right)\right)$. Note that the integral constants $A$ and $\theta$ may depend on the initial time $t_{0}$. The integration constants $A\left(t_{0}\right)$ and $\theta\left(t_{0}\right)$ will parametrize the global solution and correspond to the hydrodynamic variables which parametrize the distribution function in the local equilibrium.

The equation for $\tilde{x}_{1}$ reads

$$
\mathcal{L} \tilde{x}_{1}=-A\left(1-\frac{A^{2}}{4}\right) \sin \phi(t)+\frac{A^{3}}{4} \sin 3 \phi(t),
$$

with $\phi(t)=t+\theta_{0}\left(t_{0}\right)$. Notice that the first term in r.h.s. is a zero mode of $\mathcal{L}$, and hence the special solution to this equation contains a secular term that is expressed as a product of $t$ and a zero mode of $\mathcal{L}$. Since we have supposed that the initial value at $t=t_{0}$ is on an exact solution, we should make the corrections from the zeroth-order solution as small as possible. This condition is realized by letting the secular terms vanish at $t=t_{0}$, which is possible because we can freely add zero mode solutions to a special solution. Thus, we have the first-order solution as

$$
\begin{aligned}
\tilde{x}_{1}\left(t ; t_{0}\right)= & \left(t-t_{0}\right) \frac{A}{2}\left(1-\frac{A^{2}}{4}\right) \sin \phi(t) \\
& -\frac{A^{3}}{32} \sin 3 \phi(t),
\end{aligned}
$$

with the initial value at $t=t_{0}, W_{1}\left(t_{0}\right)=\tilde{x}_{1}\left(t_{0} ; t_{0}\right)=$ $-A^{3}\left(t_{0}\right) / 32 \cdot \sin 3 \phi\left(t_{0}\right)$. 
If we stop here, we have the perturbative solution; $\tilde{x}=$ $\tilde{x}_{0}+\epsilon \tilde{x}_{1}$, which should be valid in a local domain $t \sim t_{0}$ but becomes invalid in the global domain where $\left|t-t_{0}\right|$ can be large, due to the secular term.

We shall now take a geometrical point of view [34-36]: The function $\tilde{x}\left(t ; t_{0}\right)$ corresponds to a curve drawn in the $(t, x)$ plane for each $t_{0}$; in other words, we have a family of curves represented by $\tilde{x}\left(t ; t_{0}\right)$ in the $(t, x)$ plane; a member of the family is parametrized by $t_{0}$, and each member is close to an exact solution in the neighborhood of $t=t_{0}$. Thus, an idea is that the envelope curve of the family of curves should give a global solution. The classical theory of envelope curve says that the envelope can be constructed by solving the following equation:

$$
\left.\frac{\mathrm{d} \tilde{x}}{\mathrm{~d} t_{0}}\right|_{t_{0}=t}=0,
$$

which is called the RG equation [32,33]; here we have made an account of it on the basis of the envelope theory [34-36]. Equation (2.9) leads to the equations for $A(t)$ and $\phi(t)$,

$$
\dot{A}=\epsilon \frac{A}{2}\left(1-\frac{A^{2}}{4}\right), \quad \dot{\phi}=1,
$$

which may be called amplitude and phase equation, respectively. The original equation is reduced to these simpler equations for the amplitude and phase which parametrize the solution of the original equation. These reduced equations are readily solved, with which a resummation of the perturbation series is performed; the resumed solution is found to successfully admit a limit cycle with a radius of 2 .

The resultant envelope function as a global solution is given by

$$
\begin{aligned}
x_{\mathrm{E}}(t) & \equiv \tilde{x}(t ; t)=W(t) \\
& =A(t) \cos \phi(t)-\epsilon \frac{A^{3}(t)}{32} \sin 3 \phi(t),
\end{aligned}
$$

with $A(t)$ and $\phi(t)$ being the solution of eq. (2.10). Thus, we have succeeded in not only obtaining the asymptotic solution as a whole but also extracting the slow variables $A(t)$ and $\phi(t)$ explicitly and their governing equations.

However, there is a problem left: Does $x_{\mathrm{E}}(t) \equiv \tilde{x}(t ; t)$ indeed satisfy the original differential equation (2.1)? We give here a proof for that $[34,36]$.

First let us rewrite eq. (2.1) into a coupled equation of first order,

$$
\frac{\mathrm{d} \boldsymbol{y}(t)}{\mathrm{d} t}=\boldsymbol{F}(\boldsymbol{y}(t) ; \epsilon),
$$

where

$$
\begin{aligned}
\boldsymbol{y} & ={ }^{t}\left(q_{1}=x, q_{2}=\dot{x}\right), \\
\boldsymbol{F} & ={ }^{t}\left(q_{2},-q_{1}+\epsilon\left(1-q_{1}^{2}\right) q_{2}\right) .
\end{aligned}
$$

We have an approximate local solution to eq. (2.12) $\tilde{\boldsymbol{y}}\left(t ; t_{0}\right)$ around $t=t_{0}$ up to $O\left(\epsilon^{n}\right)$, corresponding to $\tilde{x}\left(t ; t_{0}\right)$,

$$
\frac{\mathrm{d} \tilde{\boldsymbol{y}}}{\mathrm{d} t}=\boldsymbol{F}\left(\tilde{\boldsymbol{y}}\left(t ; t_{0}\right) ; \epsilon\right)+O\left(\epsilon^{n}\right) .
$$

Now, the RG/envelope equation implies that

$$
\left.\frac{\partial \tilde{\boldsymbol{y}}\left(t ; t_{0}\right)}{\partial t_{0}}\right|_{t=t_{0}}=0 .
$$

The envelope function $\boldsymbol{y}_{\mathrm{E}}(t)$ corresponding to $x_{\mathrm{E}}(t)$ is defined by

$$
\boldsymbol{y}_{\mathrm{E}}(t)=\tilde{\boldsymbol{y}}(t ; t) .
$$

It is now easy to show that $\boldsymbol{y}_{\mathrm{E}}(t)$ satisfies eq. (2.12) for arbitrary $t$ up to the same order as $\tilde{\boldsymbol{y}}\left(t ; t_{0}\right)$ does locally. In fact, $\forall t=t_{0}$

$$
\begin{aligned}
\left.\frac{\mathrm{d} \boldsymbol{y}_{\mathrm{E}}(t)}{\mathrm{d} t}\right|_{t=t_{0}} & =\left.\frac{\mathrm{d} \tilde{\boldsymbol{y}}\left(t ; t_{0}\right)}{\mathrm{d} t}\right|_{t=t_{0}}+\left.\frac{\partial \tilde{\boldsymbol{y}}\left(t ; t_{0}\right)}{\partial t_{0}}\right|_{t=t_{0}} \\
& =\left.\frac{\mathrm{d} \tilde{\boldsymbol{y}}\left(t ; t_{0}\right)}{\mathrm{d} t}\right|_{t=t_{0}} \\
& =\boldsymbol{F}(\tilde{\boldsymbol{y}}(t ; t) ; \epsilon)+O\left(\epsilon^{n}\right) \\
& =\boldsymbol{F}\left(\boldsymbol{y}_{\mathrm{E}}(t) ; \epsilon\right)+O\left(\epsilon^{n}\right) .
\end{aligned}
$$

This completes the proof. Here eqs. (2.16) and (2.15) have been used together with the definition of $\boldsymbol{y}_{\mathrm{E}}(t)$, eq. (2.17). It should be stressed that eq. (2.18) is valid uniformly $\forall t$ i.e., in the global domain of $t$, in contrast to eq. (2.15) which is in a local domain around $t=t_{0}$.

We can summarize what we have done as follows: when there exist zero modes of the unperturbed operator, the higher-order corrections may give rise to secular terms, which are renormalized into the integral constants in the zeroth-order solution using the RG/envelope equation, and thereby, the would-be integral constants are lifted to dynamical variables.

It will be found that the would-be integral constants, $A$ and $\phi$, exactly correspond to the hydrodynamic variables characterizing the local equilibrium distribution function, such as the temperature $T$, chemical potential $\mu$, and flow velocity $u^{\mu}\left(u^{\mu} u_{\mu}=1\right)$ : Equations (2.10) of the amplitude and phase which parametrize the solution also exactly correspond to the hydrodynamic equation governing the hydrodynamic variables which parametrize the distribution function as the solution of the Boltzmann equation.

\section{Relativistic Boltzmann equation}

The relativistic Boltzmann equation (RBE) reads $[21,22]$

$$
p^{\mu} \partial_{\mu} f_{p}(x)=C[f]_{p}(x),
$$

where $f_{p}(x)$ denotes the one-particle distribution function with $p^{\mu}$ being the four-momentum of the on-shell particle, i.e., $p^{\mu} p_{\mu}=p^{2}=m^{2}$ and $p^{0}>0$, while $C[f]_{p}(x)$ in r.h.s. the collision integral

$$
\begin{aligned}
C[f]_{p}(x) \equiv & \frac{1}{2 !} \sum_{p_{1}} \frac{1}{p_{1}^{0}} \sum_{p_{2}} \frac{1}{p_{2}^{0}} \sum_{p_{3}} \frac{1}{p_{3}^{0}} \omega\left(p, p_{1} \mid p_{2}, p_{3}\right) \\
& \times\left(f_{p_{2}}(x) f_{p_{3}}(x)-f_{p}(x) f_{p_{1}}(x)\right)
\end{aligned}
$$


with $\omega\left(p, p_{1} \mid p_{2}, p_{3}\right)$ being the transition probability due to the microscopic two-particle interaction with the symmetry property

$$
\begin{aligned}
\omega\left(p, p_{1} \mid p_{2}, p_{3}\right) & =\omega\left(p_{2}, p_{3} \mid p, p_{1}\right) \\
& =\omega\left(p_{1}, p \mid p_{3}, p_{2}\right)=\omega\left(p_{3}, p_{2} \mid p_{1}, p\right),
\end{aligned}
$$

and the energy-momentum conservation

$$
\omega\left(p, p_{1} \mid p_{2}, p_{3}\right) \propto \delta^{4}\left(p+p_{1}-p_{2}-p_{3}\right) .
$$

To make the correspondence to the general formulation of the reduction theory given in $[39,37]$ explicit, we treat the momentum as a discrete variable; apart from such a formal reasoning, the summation with respect to the momentum may be interpreted as the integration in practical use as follows:

$$
\sum_{q} \equiv \int \mathrm{d}^{3} \boldsymbol{q}
$$

with $\boldsymbol{q}$ being the spatial components of the four-momentum $q^{\mu}$.

For an arbitrary vector $\varphi_{p}(x)$, the collision integral satisfies the following identity, thanks to the above-mentioned symmetry property:

$$
\begin{aligned}
& \sum_{p} \frac{1}{p^{0}} \varphi_{p}(x) C[f]_{p}(x)= \\
& \frac{1}{2 !} \frac{1}{4} \sum_{p, p_{1} \sim p_{3}} \frac{1}{p^{0} p_{1}^{0} p_{2}^{0} p_{3}^{0}} \omega\left(p, p_{1} \mid p_{2}, p_{3}\right) \\
& \quad \times\left(\varphi_{p}(x)+\varphi_{p_{1}}(x)-\varphi_{p_{2}}(x)-\varphi_{p_{3}}(x)\right) \\
& \quad \times\left(f_{p_{2}}(x) f_{p_{3}}(x)-f_{p}(x) f_{p_{1}}(x)\right) .
\end{aligned}
$$

Substituting $\left(1, p^{\mu}\right)$ into $\varphi_{p}(x)$ in eq. (3.6), we find that $\left(1, p^{\mu}\right)$ are collision invariants satisfying

$$
\sum_{p} \frac{1}{p^{0}} C[f]_{p}(x)=\sum_{p} \frac{1}{p^{0}} p^{\mu} C[f]_{p}(x)=0,
$$

due to the particle-number and energy-momentum conservation in the collision process, respectively. We note that the function $\varphi_{0 p}(x) \equiv a(x)+p^{\mu} b_{\mu}(x)$ is also a collision invariant where $a(x)$ and $b_{\mu}(x)$ are arbitrary functions of $x$. This form is, in fact, the most general form of a collision invariant [21]; see [22] for a proof.

Owing to the particle-number and energy-momentum conservation in the collision process leading to eq. (3.7), we have the balance equations for the particle current $N^{\mu}(x)$ and the energy-momentum tensor $T^{\mu \nu}(x)$,

$$
\begin{aligned}
\partial_{\mu} N^{\mu}(x) & \equiv \partial_{\mu}\left[\sum_{p} \frac{1}{p^{0}} p^{\mu} f_{p}(x)\right]=0, \\
\partial_{\nu} T^{\mu \nu}(x) & \equiv \partial_{\nu}\left[\sum_{p} \frac{1}{p^{0}} p^{\mu} p^{\nu} f_{p}(x)\right]=0,
\end{aligned}
$$

respectively. It should be noted that any dynamical properties are not contained in these equations unless the evolution of $f_{p}(x)$ has been obtained as a solution to eq. (3.1).
In the Boltzmann theory, the entropy current may be defined [21] by

$$
S^{\mu}(x) \equiv-\sum_{p} \frac{1}{p^{0}} p^{\mu} f_{p}(x)\left[\ln \left((2 \pi)^{3} f_{p}(x)\right)-1\right],
$$

where the factor $(2 \pi)^{3}$ is necessary owing to our convention (3.5) [21]. The entropy current $S^{\mu}(x)$ satisfies

$$
\partial_{\mu} S^{\mu}(x)=-\sum_{p} \frac{1}{p^{0}} C[f]_{p}(x) \ln \left((2 \pi)^{3} f_{p}(x)\right),
$$

due to eq. (3.11). One sees that $S^{\mu}(x)$ is conserved only if $\ln \left((2 \pi)^{3} f_{p}(x)\right)$ is a collision invariant, i.e., $\ln \left((2 \pi)^{3} f_{p}(x)\right)=\varphi_{0 p}(x)=a(x)+p^{\mu} b_{\mu}(x)$. One thus finds $[21,22]$ that entropy-conserving distribution function may be parametrized as

$$
f_{p}(x)=\frac{1}{(2 \pi)^{3}} \exp \left[\frac{\mu(x)-p^{\mu} u_{\mu}(x)}{T(x)}\right] \equiv f_{p}^{\mathrm{eq}}(x),
$$

with $u^{\mu}(x) u_{\mu}(x)=1$. The function (3.12) is identified with the local equilibrium distribution function called the Jüttner function [50], where $T(x), \mu(x)$, and $u^{\mu}(x)$ in eq. (3.12) are the local temperature, chemical potential, and flow velocity, respectively; see [22] for a proof. These five variables are called hydrodynamic variables. Due to the energy-momentum conservation in the collision process, we see that the collision integral identically vanishes for the local equilibrium distribution $f_{p}^{\text {eq }}(x)$ as

$$
C\left[f^{\mathrm{eq}}\right]_{p}(x)=0 .
$$

Some remarks are in order here. In the proof [22], the Gibbs-Duhem relation as given by

$$
\mathrm{d}(s / n)=\frac{1}{T}\left(\mathrm{~d}(e / n)-\frac{p}{n^{2}} \mathrm{~d} n\right),
$$

is taken for granted, where $s, e, n$, and $p$ denote the entropy, internal energy per volume, particle density, and pressure in the equilibrium state, respectively. However, Van and Biro [18] have recently argued that the conventional Gibbs-Duhem relation (3.14) may be modified so as to contain the contribution from the thermal flow in the local equilibrium state of a relativistic system, and given a different interpretation for $T(x), \mu(x)$, and $u^{\mu}(x)$ in (3.12); this modified definition of the local equilibrium state, they claim, leads to the relativistic hydrodynamic equation in the particle frame with the stable equilibrium state. Although this is certainly an interesting possibility, we will not follow this novel interpretation in this review: We shall make some comments on some related problem below.

\section{Reduction to hydrodynamic equation}

Let us try to solve the RBE (3.1) in the hydrodynamic regime, and thereby derive the hydrodynamic equations governing the hydrodynamic variables. 


\subsection{Relativistic Boltzmann equation in local rest frame} of flow velocity

To make it explicit to solve the RBE in the hydrodynamic regime, we first convert the $\mathrm{RBE}$ (3.1) into the following form with the use of the flow velocity $u^{\mu}\left(u^{\mu} u_{\mu}=1\right)$ [21],

$$
\begin{aligned}
\frac{\partial}{\partial \tau} f_{p}(\tau, \sigma)= & \frac{1}{p \cdot u} C[f]_{p}(\tau, \sigma) \\
& -\varepsilon \frac{1}{p \cdot u} p \cdot \nabla f_{p}(\tau, \sigma),
\end{aligned}
$$

where the new coordinate system $\left(\tau, \sigma^{\mu}\right)$ is defined as follows:

$$
\begin{aligned}
\frac{\partial}{\partial \tau} & =u^{\mu} \partial_{\mu} \equiv D \\
\frac{\partial}{\partial \sigma_{\mu}} & =\left(g^{\mu \nu}-u^{\mu} u^{\nu}\right) \partial_{\nu} \equiv \Delta^{\mu \nu} \partial_{\nu} \equiv \nabla^{\mu} .
\end{aligned}
$$

We note that $D$ and $\nabla^{\mu}$ are temporal and spatial differential operators familiar in the literature. In eq. (4.1), the small parameter $\varepsilon$ is introduced as a measure of the nonuniformity of the fluid, which may be identified with the Knudsen number; $\varepsilon$ will be set back to unity in the final stage of the analysis. In the present analysis based on the RG method, the perturbative expansion of the distribution function with respect to $\varepsilon$ is first performed with the zeroth order being the local equilibrium one; the dissipative effect is taken into account as a deformation of the distribution function made by the spatial inhomogeneity as the perturbation. Thus the above rewrite of the equation with $\varepsilon$ reflects the physical assumption that only the spatial inhomogeneity is the origin of the dissipation. It is noteworthy that our RG method applied to the nonrelativistic Boltzmann equation with the corresponding assumption successfully leads to the Navier-Stokes equation $[51,52]$; the present approach $[25,27]$ is simply a relativistic generalization of the nonrelativistic case.

Here we make a comment on the possibility of a rewrite of the RBE (3.1) with the use of a different time-like fourvector in place of the flow velocity $u^{\mu}$. In other words, we examine whether eq. (4.1) with $u^{\mu}$ being identified with the flow velocity is a unique rewrite of the RBE (3.1) in a covariant manner. We argue that it is the case on the basis of a physical ground.

We first introduce a generic time-like four-vector $\boldsymbol{a}^{\mu}$ with $\boldsymbol{a}^{2}>0$, and call it the macroscopic-frame vector, following $[25,53]$. Without loss of generality, the generic vector of Lorentz covariance takes the form

$$
\boldsymbol{a}^{\mu}=A_{1} u^{\mu}+A_{2} \partial^{\mu} T+A_{3} \partial^{\mu} \mu+A_{4} u^{\nu} \partial_{\nu} u^{\mu},
$$

since $u^{\mu}$ and $\partial^{\mu}$ are the only available Lorentz vectors at hand. Here, $A_{1}, A_{2}, A_{3}$, and $A_{4}$ are arbitrary functions of the temperature $T$ and the chemical potential $\mu ; A_{i}=$ $A_{i}(T, \mu)$ for $i=1,2,3,4$. Owing to the identity

$$
\partial^{\mu}=u^{\mu} u^{\nu} \partial_{\nu}+\left(g^{\mu \nu}-u^{\mu} u^{\nu}\right) \partial_{\nu}=u^{\mu} D+\nabla^{\mu},
$$

where $D$ and $\nabla^{\mu}$ have been defined in eqs. (4.2) and (4.3), eq. (4.4) is rewritten as

$$
\begin{aligned}
\boldsymbol{a}^{\mu}= & \left(A_{1}+A_{2} D T+A_{3} D \mu\right) u^{\mu} \\
& +A_{2} \nabla^{\mu} T+A_{3} \nabla^{\mu} \mu+A_{4} D u^{\mu} \\
\equiv & C_{t}(T, \mu) u^{\mu}+A_{2} \nabla^{\mu} T+A_{3} \nabla^{\mu} \mu+A_{4} D u^{\mu}
\end{aligned}
$$

with $C_{t}(T, \mu)=A_{1}+A_{2} D T+A_{3} D \mu$. The relative magnitudes of $C_{t}(T, \mu)$ and $A_{2,3,4}$ are only constrained by the inequality $\boldsymbol{a}^{2}>0$ in the present stage. However, it should be emphasized that the space-like terms with the coefficients $A_{2,3,4}$ are all derivative terms, which are supposed to be small in the hydrodynamic regime even in the dissipative regime if the dynamics is governed by the hydrodynamics at all.

By replacing $u^{\mu}$ by $\boldsymbol{a}^{\mu}$ given by (4.6), we have the generic coordinate system $\left(\tilde{\tau}, \tilde{\sigma}^{\mu}\right)$ as defined by

$$
\begin{aligned}
\frac{\partial}{\partial \tilde{\tau}} & \equiv \frac{\boldsymbol{a}^{\mu}}{\boldsymbol{a}^{2}} \partial_{\mu}, \\
\frac{\partial}{\partial \tilde{\sigma}_{\mu}} & \equiv\left(g^{\mu \nu}-\frac{\boldsymbol{a}^{\mu} \boldsymbol{a}^{\nu}}{\boldsymbol{a}^{2}}\right) \partial_{\nu} .
\end{aligned}
$$

With this coordinate system, the RBE is rewritten as

$$
\begin{aligned}
\frac{\partial}{\partial \tilde{\tau}} f_{p}(\tilde{\tau}, \tilde{\sigma})= & \frac{1}{p \cdot \boldsymbol{a}} C[f]_{p}(\tilde{\tau}, \tilde{\sigma}) \\
& -\varepsilon \frac{1}{p \cdot \boldsymbol{a}} p^{\mu} \frac{\partial}{\partial \tilde{\sigma}^{\mu}} f_{p}(\tilde{\tau}, \tilde{\sigma}),
\end{aligned}
$$

where the $\varepsilon$ is again multiplied to $\partial / \partial \tilde{\sigma}^{\mu}$ as was done in eq. (4.1) where it is supposed that only the spatial inhomogeneity is the origin of the dissipation. Then the space-like terms with the coefficients $A_{2}$ and $A_{3}$ in $\boldsymbol{a}^{\mu}$ are of higher order with respect to $\varepsilon$ and should be ignored in this set up. Furthermore, since we start with a stationary solution with vanishing time dependence in the $\mathrm{RG}$ approach, the term with $A_{4}$ should be also ignored. Thus we have

$$
\boldsymbol{a}^{\mu}=C_{t}(T, \mu) u^{\mu},
$$

and, accordingly,

$$
\frac{\partial}{\partial \tilde{\sigma}_{\mu}}=\nabla^{\mu} .
$$

If we naturally require that $C_{t}(T, \mu)$ should be independent of the momentum $p^{\mu}$, it is easy to show [54] that the "normalization" factor $C_{t}(T, \mu)$ can be made unity without loss of generality, in conformity of the natural choice $[21,22] \boldsymbol{a}^{\mu}=u^{\mu}$.

It is remarkable that this natural choice uniquely leads to the hydrodynamic equation in the energy (LandauLifshitz) frame, as will be shown and discussed later [25, 53]. Conversely, a choice of $C_{t}(T, \mu)$ different from unity with a momentum dependence could lead to various hydrodynamic equations other than that of Landau and Lifshitz, including the one in the particle frame for viscous fluids as was shown by the present authors [25, 53]. However, it is worth emphasizing that the particle frame can be realized only when $C_{t}(T, \mu)$ has a peculiar momentum dependence such as $C_{t}(T, \mu)=m /(p \cdot u)$ 
$\left(\boldsymbol{a}^{\mu}=(m /(p \cdot u)) u^{\mu}\right)[25,53]$. In retrospect, however, the possible momentum dependence of $C_{t}(T, \mu)$ cannot be legitimate for $\boldsymbol{a}^{\mu}$ to play a macroscopic-frame vector, because it means that the covariant and macroscopic spacetime in the particle frame is defined for a respective particle state with a definite energy momentum, which is certainly unnatural and lead to a trouble in a physical interpretation [54]. Thus, we naturally require that $C_{t}(T, \mu)$ is independent of the momentum $p^{\mu}$ and hence $\boldsymbol{a}^{\mu}=u^{\mu}$.

\subsection{Hydrodynamics from relativistic Boltzmann equation by the renormalization-group method}

Applying the perturbation theory to eq. (4.1), we derive the relativistic dissipative hydrodynamic equation as the infrared asymptotic dynamics of the RBE by the RG method [25,27].

In this approach, we first try to obtain the perturbative solution $\tilde{f}_{p}$ to eq. (4.1) around the arbitrary initial time $\tau=\tau_{0}$ with the initial value $f_{p}\left(\tau_{0}, \sigma\right)$,

$$
\tilde{f}_{p}\left(\tau=\tau_{0}, \sigma ; \tau_{0}\right)=f_{p}\left(\tau_{0}, \sigma\right) .
$$

Note that the solution depends on the initial time $\tau_{0}$ at which $\tilde{f}_{p}\left(\tau=\tau_{0}, \sigma ; \tau_{0}\right)$ is supposed to be on an exact solution. We expand the initial value as well as the solution with respect to $\varepsilon$ as follows:

$$
\begin{aligned}
\tilde{f}_{p}\left(\tau, \sigma ; \tau_{0}\right)= & \tilde{f}_{p}^{(0)}\left(\tau, \sigma ; \tau_{0}\right)+\varepsilon \tilde{f}_{p}^{(1)}\left(\tau, \sigma ; \tau_{0}\right) \\
& +\varepsilon^{2} \tilde{f}_{p}^{(2)}\left(\tau, \sigma ; \tau_{0}\right)+\ldots \\
f_{p}\left(\tau_{0}, \sigma\right)= & f_{p}^{(0)}\left(\tau_{0}, \sigma\right)+\varepsilon f_{p}^{(1)}\left(\tau_{0}, \sigma\right) \\
& +\varepsilon^{2} \tilde{f}_{p}^{(2)}\left(\tau, \sigma ; \tau_{0}\right)+\ldots
\end{aligned}
$$

The zeroth-order equation reads

$$
\frac{\partial}{\partial \tau} \tilde{f}_{p}^{(0)}\left(\tau, \sigma ; \tau_{0}\right)=\frac{1}{p \cdot u} C\left[\tilde{f}^{(0)}\right]_{p}\left(\tau, \sigma ; \tau_{0}\right) .
$$

Since we are looking for the slow motion to be realized asymptotically when $\tau \rightarrow \infty$, we take the stationary solution satisfying

$$
\frac{\partial}{\partial \tau} \tilde{f}_{p}^{(0)}\left(\tau, \sigma ; \tau_{0}\right)=0
$$

implying that $C\left[\tilde{f}^{(0)}\right]_{p}\left(\tau, \sigma ; \tau_{0}\right)=0 \forall \sigma$, which is solved by a local equilibrium distribution function, i.e., the Jüttner distribution function,

$$
\begin{aligned}
\tilde{f}_{p}^{(0)}\left(\tau, \sigma ; \tau_{0}\right) & =\frac{1}{(2 \pi)^{3}} \exp \left[\frac{\mu\left(\sigma ; \tau_{0}\right)-p^{\mu} u_{\mu}\left(\sigma ; \tau_{0}\right)}{T\left(\sigma ; \tau_{0}\right)}\right] \\
& \equiv f_{p}^{\text {eq }}\left(\sigma ; \tau_{0}\right)
\end{aligned}
$$

with $u^{\mu}\left(\sigma ; \tau_{0}\right) u_{\mu}\left(\sigma ; \tau_{0}\right)=1$. Here the would-be integration constants $T\left(\sigma ; \tau_{0}\right), \mu\left(\sigma ; \tau_{0}\right)$, and $u_{\mu}\left(\sigma ; \tau_{0}\right)$ are independent of $\tau$ but may depend on $\tau_{0}$ as well as $\sigma$.
Now that the zeroth-order solution is given, the firstorder equation reads

$$
\frac{\partial}{\partial \tau} \tilde{f}_{p}^{(1)}(\tau)=\sum_{q} A_{p q} \tilde{f}_{q}^{(1)}(\tau)+F_{p},
$$

with

$$
F_{p} \equiv-\frac{1}{p \cdot u} p \cdot \nabla f_{p}^{\mathrm{eq}}
$$

where $A_{p q}$ denotes a matrix element of the linearized collision operator $A$, i.e.,

$$
(A)_{p q}=\left.A_{p q} \equiv \frac{1}{p \cdot u} \frac{\partial}{\partial f_{q}} C[f]_{p}\right|_{f=f \text { eq }} .
$$

Let us examine the spectral properties of $A$; for which, it is found convenient to convert $A$ to another linear operator,

$$
L \equiv\left(f^{\mathrm{eq}}\right)^{-1} A f^{\mathrm{eq}},
$$

with the diagonal matrix $\left(f^{\mathrm{eq}}\right)_{p q} \equiv f_{p}^{\mathrm{eq}} \delta_{p q}$. Next we define an inner product between arbitrary non-zero vectors $\varphi$ and $\psi$ by

$$
\langle\varphi, \psi\rangle \equiv \sum_{p} \frac{1}{p^{0}}(p \cdot u) f_{p}^{\mathrm{eq}} \varphi_{p} \psi_{p},
$$

which satisfies the positive definiteness of the norm as

$$
\langle\varphi, \varphi\rangle=\sum_{p} \frac{1}{p^{0}}(p \cdot u) f_{p}^{\mathrm{eq}}\left(\varphi_{p}\right)^{2}>0,
$$

for $\varphi_{p} \neq 0$, since both $p^{\mu}$ and $u^{\mu}$ are time-like vectors with $p^{0}>0$.

Then it can be shown $[25,27]$ that the linearized collision operator $L$ has remarkable properties that it is seminegative definite and has five zero modes given by

$$
\varphi_{0 p}^{\alpha} \equiv \begin{cases}p^{\mu} & \text { for } \alpha=\mu \\ 1 \times m & \text { for } \alpha=4\end{cases}
$$

The functional subspace spanned by the five zero modes is called the $\mathrm{P}_{0}$ space and the projection operator to it is denoted by $P_{0}$,

$$
\left[P_{0} \psi\right]_{p} \equiv \varphi_{0 p}^{\alpha} \eta_{0 \alpha \beta}^{-1}\left\langle\varphi_{0}^{\beta}, \psi\right\rangle,
$$

where $\eta_{0 \alpha \beta}^{-1}$ is the inverse matrix of the the $\mathrm{P}_{0}$ space metric matrix $\eta_{0}^{\alpha \beta}$ defined by

$$
\eta_{0}^{\alpha \beta} \equiv\left\langle\varphi_{0}^{\alpha}, \varphi_{0}^{\beta}\right\rangle
$$

We also call the complement to $\mathrm{P}_{0}$ the $\mathrm{Q}_{0}$ space and introduce $Q_{0} \equiv 1-P_{0}$. In the following, we also use the modified projection operators defined by

$$
\bar{P}_{0}=f^{\mathrm{eq}} P_{0}\left(f^{\mathrm{eq}}\right)^{-1}, \quad \bar{Q}_{0}=f^{\mathrm{eq}} Q_{0}\left(f^{\mathrm{eq}}\right)^{-1},
$$

which means, for example,

$$
\left[\bar{P}_{0} \psi\right]_{p}=f_{p}^{\text {eq }} \varphi_{0 p}^{\alpha} \eta_{0 \alpha \beta}^{-1}\left\langle\varphi_{0}^{\beta},\left(f^{\text {eq }}\right)^{-1} \psi\right\rangle .
$$



reads

Then the perturbative solution up to the second order

$$
\begin{aligned}
\tilde{f}_{p}\left(\tau, \sigma ; \tau_{0}\right)= & \tilde{f}_{p}^{(0)}\left(\tau, \sigma ; \tau_{0}\right)+\varepsilon \tilde{f}_{p}^{(1)}\left(\tau, \sigma ; \tau_{0}\right) \\
& +\varepsilon^{2} \tilde{f}_{p}^{(2)}\left(\tau, \sigma ; \tau_{0}\right)+O\left(\varepsilon^{3}\right),
\end{aligned}
$$

where $\tilde{f}^{(1)}\left(\tau, \sigma ; \tau_{0}\right)=\left(\tau-\tau_{0}\right) \bar{P}_{0} F-A^{-1} \bar{Q}_{0} F$ and a lengthy formula for $\tilde{f}^{(2)}\left(\tau, \sigma ; \tau_{0}\right)$, which we do not write down for the sake of space; see [27] for the details.

We remark that this solution contains secular terms, which apparently invalidates the perturbative expansion for $\tau$ away from the initial time $\tau_{0}$. We can, however, utilize the secular terms to obtain an asymptotic solution valid in a global domain [34-37]. Indeed we have a family of curves $\tilde{f}_{p}\left(\tau, \sigma ; \tau_{0}\right)$ parameterized with $\tau_{0}$ : They are all on the exact solution $f_{p}(\sigma ; \tau)$ at $\tau=\tau_{0}$ up to $O\left(\varepsilon^{3}\right)$, although only valid for $\tau$ near $\tau_{0}$ locally. Then, the envelope curve of the family of curves, which is in contact with each local solution at $\tau=\tau_{0}$, will give a global solution in our asymptotic situation, which is shown to be the case [34-37]. According to the classical theory of envelopes, the envelope that is in contact with any curve in the family at $\tau=\tau_{0}$ is obtained [34-36] by

$$
\left.\frac{\mathrm{d}}{\mathrm{d} \tau_{0}} \tilde{f}_{p}\left(\tau, \sigma ; \tau_{0}\right)\right|_{\tau_{0}=\tau}=0
$$

The derivative w.r.t. $\tau_{0}$ hits the hydrodynamic variables, and hence we have the evolution equation of them that is identified with the hydrodynamic equation $[51,52,25]$. We also note that the invariant manifold which corresponds to the hydrodynamics in the functional space of the distribution function is explicitly obtained as an envelope function $[25,27]: f_{\mathrm{E} p}(\tau, \sigma)=\tilde{f}_{p}\left(\tau, \sigma ; \tau_{0}=\tau\right)$, the explicit form of which is referred to $[25,27]$. We note that this solution is valid in a global domain of time in the asymptotic region [27].

Putting back $\varepsilon$ to 1 , eq. (4.30) is reduced to the following form in this approximation:

$$
\sum_{p} \frac{1}{p^{0}} \varphi_{0 p}^{\alpha}\left[(p \cdot u) \frac{\partial}{\partial \tau}+p \cdot \nabla\right]\left(f_{p}^{\mathrm{eq}}+\delta f_{p}^{(1)}\right)=0
$$

where $\delta f_{p}^{(1)}$ denotes the first-order correction to the distribution function

$$
\delta f_{p}^{(1)} \equiv-\left[A^{-1} \bar{Q}_{0} F\right]_{p}
$$

If one uses the identity $(p \cdot u) \partial / \partial \tau+p \cdot \nabla=p^{\mu} \partial_{\mu}$, eq. (4.31) is found to have the following form:

$$
\partial_{\mu} T_{1 \mathrm{st}}^{\mu \nu}=0, \quad \partial_{\mu} N_{1 \mathrm{st}}^{\mu}=0,
$$

with $T_{1 \mathrm{st}}^{\mu \nu}=T^{(0) \mu \nu}+\delta T_{1 \mathrm{st}}^{\mu \nu}$ and $N_{1 \mathrm{st}}^{\mu}=N^{(0) \mu}+\delta N_{1 \mathrm{st}}^{\mu}$. Here,

$$
\begin{aligned}
T^{(0) \mu \nu} & \equiv \sum_{p} \frac{1}{p^{0}} p^{\mu} p^{\nu} f_{p}^{\mathrm{eq}}=e u^{\mu} u^{\nu}-p \Delta^{\mu \nu}, \\
N^{(0) \mu} & \equiv \sum_{p} \frac{1}{p^{0}} p^{\mu} f_{p}^{\mathrm{eq}}=n u^{\mu},
\end{aligned}
$$

with $e, p$, and $n$ being the internal energy, pressure, and particle number density for the relativistic ideal gas, respectively, while the dissipative parts are given as a deviation of the local equilibrium distribution function

$$
\begin{aligned}
\delta T_{1 \mathrm{st}}^{\mu \nu} & \equiv \sum_{p} \frac{1}{p^{0}} p^{\mu} p^{\nu} \delta f_{p}^{(1)}, \\
\delta N_{1 \mathrm{st}} & \equiv \sum_{p} \frac{1}{p^{0}} p^{\mu} \delta f_{p}^{(1)} .
\end{aligned}
$$

As is well known, the local equilibrium distribution function as given by (4.17) only gives the relativistic Euler equation without dissipation.

\subsection{Possible uniqueness of Landau-Lifshitz frame}

In this subsection, we present the explicit form of the dissipative parts $\delta T_{1 \mathrm{st}}^{\mu \nu}$ and $\delta N_{1 \mathrm{st}}^{\mu}$ and discuss their properties. An evaluation of eq. (4.36) together with (4.32) gives [25, 27],

$$
\begin{aligned}
\delta T_{1 \mathrm{st}}^{\mu \nu} & =\zeta \Delta^{\mu \nu} \nabla \cdot u+2 \eta \Delta^{\mu \nu \rho \sigma} \nabla_{\rho} u_{\sigma} \\
\delta N_{1 \mathrm{st}}^{\mu} & =\lambda \frac{1}{\hat{h}^{2}} \nabla^{\mu} \frac{\mu}{T}
\end{aligned}
$$

respectively, with $\Delta^{\mu \nu \rho \sigma} \equiv 1 / 2 \cdot\left(\Delta^{\mu \rho} \Delta^{\nu \sigma}+\Delta^{\mu \sigma} \Delta^{\nu \rho}-2 / 3\right.$. $\left.\Delta^{\mu \nu} \Delta^{\rho \sigma}\right)$. Here, $\hat{h}$ denotes the reduced enthalpy per particle. The bulk and shear viscosities and the thermal conductivity are denoted by $\zeta, \eta$ and $\lambda$, respectively. It is clear that these formulas completely agree with those proposed by Landau and Lifshitz [17]. Indeed, the respective dissipative parts $\delta T_{1 \text { st }}^{\mu \nu}$ and $\delta N_{1 \text { st }}^{\mu}$ in eqs. (4.38) and (4.39) meet Landau and Lifshitz's ansatz,

$$
\begin{aligned}
\delta e & \equiv u_{\mu} \delta T_{1 \mathrm{st}}^{\mu \nu} u_{\nu}=0, \\
\delta n & \equiv u_{\mu} \delta N_{1 \mathrm{st}}^{\mu}=0, \\
Q_{\mu} & \equiv \Delta_{\mu \nu} \delta T_{1 \mathrm{st}}^{\nu \rho} u_{\rho}=0 .
\end{aligned}
$$

Thus we find that the frame on which the flow velocity is defined inevitably becomes the Landau-Lifshitz (energy) frame, if the hydrodynamics is to be consistent with the underlying relativistic Boltzmann equation ${ }^{1}$.

Let us see the above fact in the level of the distribution function. We first note that eqs. (4.36) and (4.37) can be rewritten as

$$
\begin{aligned}
\delta T_{1 \mathrm{st}}^{\mu \nu} & =\sum_{p} \frac{1}{p^{0}} p^{\mu} p^{\nu} f_{p}^{\mathrm{eq}} \bar{\phi}_{p} \\
\delta N_{1 \mathrm{st}} & =\sum_{p} \frac{1}{p^{0}} p^{\mu} f_{p}^{\mathrm{eq}} \bar{\phi}_{p}
\end{aligned}
$$

with

$$
\bar{\phi}_{p}=-\left[L^{-1} Q_{0}\left(f^{\mathrm{eq}}\right)^{-1} F\right]_{p},
$$

${ }^{1}$ The uniqueness of the energy frame for the relativistic hydrodynamics is recently argued also in a different context [55]. 
which belongs to the $\mathrm{Q}_{0}$ space and thus orthogonal to the zero modes,

$$
\left\langle\varphi_{0}^{\alpha}, \bar{\phi}\right\rangle=0 \quad \text { for } \quad \alpha=0,1,2,3,4 .
$$

Here, the inner product is defined by eq. (4.22). Then, eq. (4.46), with $\alpha=\mu$, is reduced to

$$
\begin{aligned}
0 & =\sum_{p} \frac{1}{p^{0}}(p \cdot u) f_{p}^{\mathrm{eq}} p^{\mu} \bar{\phi}_{p}=u_{\nu} \sum_{p} \frac{1}{p^{0}} p^{\nu} p^{\mu} f_{p}^{\mathrm{eq}} \bar{\phi}_{p} \\
& =u_{\nu} \delta T_{1 \mathrm{st}}^{\mu \nu} .
\end{aligned}
$$

Similarly, eq. (4.46) with $\alpha=4$ is reduced to $u_{\mu} \delta N_{1 \text { st }}^{\mu}=$ 0 . Thus, one can readily see that these equations coincide with Landau and Lifshitz's ansatz: We remark that eq. (4.47) implies the following two equations: $\delta e \equiv$ $u_{\mu} u_{\nu} \delta T_{1 \mathrm{st}}^{\mu \nu}=0$ and $Q_{\rho} \equiv \Delta_{\rho \mu} u_{\nu} \delta T_{1 \mathrm{st}}^{\mu \nu}=0$, which are nothing but the matching conditions [21] imposed to select the energy frame in all the other existing approaches based on the Boltzmann equation. In other words, we have given the foundation to the matching conditions [21] for the energy frame.

A remark is in order here. The uniqueness of the energy frame comes from the two natural conditions used in the derivation, i.e., the identification of the time-like vector $u^{\mu}$ in the Jüttner distribution function (4.17) with the flow velocity and the physical assumption that the dissipative effect comes from only the spatial inhomogeneity. If one of these conditions were to be challenged, as claimed in [18], for instance, the uniqueness of the energy frame could be violated. It is clear that further studies are needed for establishing the uniqueness of the energy frame in the relativistic hydrodynamics for viscous fluids.

\subsection{Transport coefficients}

Since our theory starts from a microscopic theory as statistical mechanics, we have the microscopic expressions for the transport coefficients appearing in the hydrodynamic tensor (4.38) and current (4.39), as follows:

$$
\begin{aligned}
\zeta & =-\frac{1}{T}\left\langle\tilde{\Pi}, L^{-1} \tilde{\Pi}\right\rangle, \\
\lambda & =\frac{1}{3 T^{2}}\left\langle\tilde{J}^{\mu}, L^{-1} \tilde{J}_{\mu}\right\rangle, \\
\eta & =-\frac{1}{10 T}\left\langle\tilde{\pi}^{\mu \nu}, L^{-1} \tilde{\pi}_{\mu \nu}\right\rangle .
\end{aligned}
$$

Here, we have introduced the following microscopic thermal forces $\left(\tilde{\Pi}_{p}, \tilde{J}_{p}^{\mu}, \tilde{\pi}_{p}^{\mu \nu}\right) \equiv\left(\Pi_{p}, J_{p}^{\mu}, \pi_{p}^{\mu \nu}\right) /(p \cdot u)$, with

$$
\begin{aligned}
\Pi_{p} \equiv & (4 / 3-\gamma)(p \cdot u)^{2}+((\gamma-1) T \hat{h}-\gamma T)(p \cdot u) \\
& -1 / 3 \cdot m^{2} \\
J_{p}^{\mu} \equiv & -((p \cdot u)-T \hat{h}) \Delta^{\mu \nu} p_{\nu} \\
\pi_{p}^{\mu \nu} \equiv & \Delta^{\mu \nu \rho \sigma} p_{\rho} p_{\sigma} .
\end{aligned}
$$

Here, $\gamma$ denotes the ratio of the constant pressure and volume heat capacities. We note that the microscopic expressions for the transport coefficients (4.48)-(4.50) are in agreement with those given by the Chapman-Enskog method [21].

It is noteworthy that the transport coefficients can be rewritten in the Green-Kubo formula [29]. With the use of the "time-dependent thermal force" defined by

$$
\tilde{\Pi}_{p}(s) \equiv \sum_{q}\left[e^{s L}\right]_{p q} \tilde{\Pi}_{q}
$$

and so on, the relaxation functions are given by the time correlators

$$
R_{\zeta}(s) \equiv \frac{1}{T}\langle\tilde{\Pi}(0), \tilde{\Pi}(s)\rangle,
$$

and so on for $R_{\lambda}(s)$ and $R_{\eta}(s)$ with obvious modifications to $R_{\zeta}(s)$. Then the transport coefficients given in eqs. (4.48)-(4.50) are rewritten as follows $[25,27]$ :

$$
\begin{aligned}
\zeta & =\int_{0}^{\infty} \mathrm{d} s R_{\zeta}(s), \quad \lambda=\int_{0}^{\infty} \mathrm{d} s R_{\lambda}(s), \\
\eta & =\int_{0}^{\infty} \mathrm{d} s R_{\eta}(s) .
\end{aligned}
$$

\section{Generic stability of relativistic hydrodynamic equation in energy frame}

In this section, we shall provide a proof [27] that generic constant solutions of the relativistic dissipative hydrodynamic equation in the energy frame is stable against a small perturbation [56], on account of the positive definiteness of the inner product as shown in eq. (4.23).

For this purpose, we first note that $F_{p}$ is reduced to

$$
F_{p}=-f_{p}^{\mathrm{eq}} \frac{1}{p \cdot u} p^{\mu} \varphi_{0 p}^{\alpha} \nabla_{\mu} X_{\alpha}
$$

with $\varphi_{0 p}^{\alpha}$ being the zero modes defined in (4.24) and

$$
X_{\alpha} \equiv \begin{cases}-u_{\nu} / T & \text { for } \alpha=\nu \\ m^{-1} \mu / T & \text { for } \alpha=4\end{cases}
$$

Then eq. (4.31) is rewritten in the following form:

$$
\begin{aligned}
& \sum_{p} \frac{1}{p^{0}} \varphi_{0 p}^{\alpha}\left[(p \cdot u) \frac{\partial}{\partial \tau}+p \cdot \nabla\right] \\
& \quad \times\left[f_{p}^{\mathrm{eq}}\left(1+\left[L^{-1} \varphi_{1}^{\nu \beta}\right]_{p} \nabla_{\nu} X_{\beta}\right)\right]=0,
\end{aligned}
$$

where

$$
\varphi_{1 p}^{\mu \alpha} \equiv\left[Q_{0} \tilde{\varphi}_{1}^{\mu \alpha}\right]_{p},
$$

with $\tilde{\varphi}_{1 p}^{\mu \alpha} \equiv p^{\mu} \varphi_{0 p}^{\alpha} /(p \cdot u)$.

Now, a generic constant solution means that it describes a system having a finite homogeneous flow with a constant temperature and a constant chemical potential, as follows:

$$
T(\sigma ; \tau)=T_{0}, \quad \mu(\sigma ; \tau)=\mu_{0}, \quad u_{\mu}(\sigma ; \tau)=u_{0 \mu},
$$


where $T_{0}, \mu_{0}$, and $u_{0 \mu}$ are constant. We note that these states include the thermal equilibrium state as a special case.

We shall show the linear stability of the constant solution of the relativistic dissipative hydrodynamic equation in the energy frame. We represent $T, \mu$, and $u_{\mu}$ around the constant solution as follows:

$$
\begin{aligned}
T(\sigma ; \tau) & =T_{0}+\delta T(\sigma ; \tau), \\
\mu(\sigma ; \tau) & =\mu_{0}+\delta \mu(\sigma ; \tau), \\
u_{\mu}(\sigma ; \tau) & =u_{0 \mu}+\delta u_{\mu}(\sigma ; \tau),
\end{aligned}
$$

where the deviations $\delta T, \delta \mu$, and $\delta u_{\mu}$ are assumed to so small that terms in the second or higher orders of them can be neglected. Instead of these six variables which are not independent of each other because $\delta u_{\mu} u_{0}^{\mu}=0$, we use the following five independent variables:

$$
\delta X_{\alpha} \equiv \begin{cases}-\delta\left(\frac{u_{\mu}}{T}\right)=-\frac{\delta u_{\mu}}{T_{0}}+\delta T \frac{u_{0 \mu}}{T_{0}^{2}} & \text { for } \alpha=\mu, \\ m^{-1} \delta\left(\frac{\mu}{T}\right)=m^{-1}\left(\frac{\delta \mu}{T_{0}}-\delta T \frac{\mu_{0}}{T_{0}^{2}}\right) & \text { for } \alpha=4\end{cases}
$$

Substituting eq. (5.9) into eq. (5.3) and with some manipulation, we obtain the linearized equation governing $\delta X_{\alpha}$ as

$$
\begin{aligned}
& \left(\left\langle\varphi_{0}^{\alpha}, \varphi_{0}^{\beta}\right\rangle+\left\langle\varphi_{0}^{\alpha}, L^{-1} \varphi_{1}^{\nu \beta}\right\rangle \nabla_{\nu}\right) \frac{\partial}{\partial \tau} \delta X_{\beta} \\
& +\left(\left\langle\tilde{\varphi}_{1}^{\mu \alpha}, \varphi_{0}^{\beta}\right\rangle \nabla_{\mu}+\left\langle\tilde{\varphi}_{1}^{\mu \alpha}, L^{-1} \varphi_{1}^{\nu \beta}\right\rangle \nabla_{\mu} \nabla_{\nu}\right) \delta X_{\beta}=0 .
\end{aligned}
$$

Here, we have used the following simple relation:

$$
\delta\left(f_{p}^{\mathrm{eq}}\right)=f_{p}^{\mathrm{eq}} \varphi_{0 p}^{\alpha} \delta X_{\alpha} .
$$

We note that all the coefficients in eq. (5.10) take constant values because they are solely given by the constant solution $\left(T, \mu, u_{\mu}\right)=\left(T_{0}, \mu_{0}, u_{0 \mu}\right)$. Owing to the orthogonality between the $\mathrm{P}_{0}$ and $\mathrm{Q}_{0}$ spaces, eq. (5.10) is reduced to

$$
\eta_{0}^{\alpha \beta} \frac{\partial}{\partial \tau} \delta X_{\beta}+B^{\alpha \beta} \delta X_{\beta}=0
$$

Here $\eta_{0}^{\alpha \beta}$ is the metric tensor defined in (4.26) and $B^{\alpha \beta}$ is defined by

$$
B^{\alpha \beta} \equiv\left\langle\tilde{\varphi}_{1}^{\mu \alpha}, \varphi_{0}^{\beta}\right\rangle \nabla_{\mu}+\eta_{1}^{\mu \alpha \nu \beta} \nabla_{\mu} \nabla_{\nu},
$$

with

$$
\eta_{1}^{\mu \alpha \nu \beta} \equiv\left\langle\varphi_{1}^{\mu \alpha}, L^{-1} \varphi_{1}^{\nu \beta}\right\rangle
$$

Both $\eta_{0}$ and $B$ are symmetric tensors.

With the ansatz $\delta X_{\alpha}(\sigma ; \tau)=\delta \tilde{X}_{\alpha}(k ; \Lambda) \mathrm{e}^{i k \cdot \sigma-\Lambda \tau}$ eq. (5.12) leads to the following algebraic equation:

$$
\left(\Lambda \eta_{0}^{\alpha \beta}-\tilde{B}^{\alpha \beta}\right) \delta \tilde{X}_{\beta}=0
$$

with $\tilde{B}^{\alpha \beta} \equiv i\left\langle\tilde{\varphi}_{1}^{\mu \alpha}, \varphi_{0}^{\beta}\right\rangle k_{\mu}-\eta_{1}^{\mu \alpha \nu \beta} k_{\mu} k_{\nu}$. Thus we have the eigenvalue equation as follows:

$$
\operatorname{det}\left(\Lambda \eta_{0}-\tilde{B}\right)=0
$$

which leads to the dispersion relation $\Lambda=\Lambda(k)$. The stability of the generic constant solution (5.5) against a small perturbation is assured when the real part of $\Lambda(k)$ is nonnegative for any $k^{\mu}$, which is shown to be the case as follows.

Now, recall that the metric matrix $\eta_{0}$ is a real symmetric and positive-definite matrix, which implies that it has a Cholesky decomposition,

$$
\eta_{0}^{-1}={ }^{t} U U
$$

where $U$ denotes a real matrix and ${ }^{t} U$ a transposed matrix of $U$. Then eq. (5.16) is converted to

$$
\operatorname{det}\left(\Lambda I-U \tilde{B}^{t} U\right)=0
$$

where $I$ denotes the unit matrix. Equation (5.18) tells us that $\Lambda(k)$ is an eigen value of $U \tilde{B}^{t} U$.

There is the following theorem: The real part of the eigen value of a complex matrix $C$ is non-negative when the Hermite matrix $\operatorname{Re}(C) \equiv\left(C+C^{\dagger}\right) / 2$ is semi-positive definite. Applying this theorem to the present case, we find that the real part of $\Lambda(k)$ becomes nonnegative for any $k^{\mu}$ when $\operatorname{Re}\left(U \tilde{B}^{t} U\right)$ is a semi-positive definite matrix, which is shown to be the case, as follows:

$$
\begin{aligned}
& w_{\alpha}\left[\operatorname{Re}\left(U \tilde{B}^{t} U\right)\right]^{\alpha \beta} w_{\beta}= \\
& w_{\alpha}\left[U \operatorname{Re}(\tilde{B})^{t} U\right]^{\alpha \beta} w_{\beta}= \\
& {[w U]_{\alpha}[\operatorname{Re}(\tilde{B})]^{\alpha \beta}[w U]_{\beta}=} \\
& -[w U]_{\alpha} \eta_{1}^{\mu \alpha \nu \beta} k_{\mu} k_{\nu}[w U]_{\beta}= \\
& -\left\langle k_{\mu}[w U]_{\alpha} \varphi_{1}^{\mu \alpha}, L^{-1} k_{\nu}[w U]_{\beta} \varphi_{1}^{\nu \beta}\right\rangle= \\
& -\left\langle\psi, L^{-1} \psi\right\rangle \geq 0 \text { for } w_{\alpha} \neq 0,
\end{aligned}
$$

with $\psi_{p} \equiv k_{\mu}[w U]_{\alpha} \varphi_{1 p}^{\mu \alpha}$. This completes the proof that the generic constant solution in eq. (5.5) is stable against a small perturbation.

\section{Second-order equations and moment method}

In the first-order hydrodynamic equations, the zero modes of the linearized collision operator form the invariant manifold on which hydrodynamics is defined; the would-be constant zero modes acquire the time dependence on the manifold by the RG equation. Our formalism can be extended so as to incorporate excited modes as additional components of the invariant/attractive manifold [40], and 
hence we can derive an extended thermodynamics or Israel-Stewart type equation with novel microscopic expressions of the relaxation times and lengths [40]. Furthermore, our theory suggests a proper ansatz for the distribution function to be used in the moment method [40]. For shortage of space, we here give a sketch of some of our results for the extended thermodynamics, leaving the detailed derivation in a separate paper [40]. We emphasize that our theory gives an explicit construction of the invariant manifold corresponding to thirteen moments, which has been long sought for [42-47].

\subsection{A brief review of Grad's thirteen-moment method and Grad-Müller equation: non-relativistic case}

In Grad's thirteen-moment method [49,21], the one-particle distribution function $f_{\boldsymbol{v}}(t, \boldsymbol{x})$ is represented as

$$
f_{\boldsymbol{v}}(t, \boldsymbol{x})=f_{\boldsymbol{v}}^{\mathrm{eq}}(t, \boldsymbol{x})\left(1+\Phi_{\boldsymbol{v}}(t, \boldsymbol{x})\right),
$$

where $f_{\boldsymbol{v}}^{\text {eq }}$ denotes the Maxwell distribution function and $\Phi_{\boldsymbol{v}}$ the deviation from $f_{\boldsymbol{v}}^{\text {eq }}$ given by

$$
\begin{aligned}
\Phi_{\boldsymbol{v}}(t, \boldsymbol{x}) & =\hat{\pi}_{\boldsymbol{v}}^{i j}(t, \boldsymbol{x}) \pi^{i j}(t, \boldsymbol{x})+\hat{J}_{\boldsymbol{v}}^{i}(t, \boldsymbol{x}) J^{i}(t, \boldsymbol{x}) \\
& \equiv \Phi_{\boldsymbol{v}}^{\mathrm{G}}(t, \boldsymbol{x}),
\end{aligned}
$$

with

$$
\begin{aligned}
\hat{\pi}_{\boldsymbol{v}}^{i j}(t, \boldsymbol{x}) & \equiv m\left(\delta v^{i}(t, \boldsymbol{x}) \delta v^{j}(t, \boldsymbol{x})-\frac{1}{3} \delta^{i j}|\boldsymbol{\delta} \boldsymbol{v}(t, \boldsymbol{x})|^{2}\right) \\
\hat{J}_{\boldsymbol{v}}^{i}(t, \boldsymbol{x}) & \equiv\left(\frac{m}{2}|\boldsymbol{\delta} \boldsymbol{v}(t, \boldsymbol{x})|^{2}-\frac{5}{2} T(t, \boldsymbol{x})\right) \delta v^{i}(t, \boldsymbol{x}) .
\end{aligned}
$$

Here, $\boldsymbol{\delta} \boldsymbol{v}(t, \boldsymbol{x}) \equiv \boldsymbol{v}-\boldsymbol{u}(t, \boldsymbol{x})$ is the peculiar velocity.

Then the evolution equation of the thirteen coefficients are determined by the equations all of which are derived from the Boltzmann equation $(\partial / \partial t+\boldsymbol{v} \cdot \boldsymbol{\nabla}) f_{\boldsymbol{v}}(t, \boldsymbol{x})=$ $C[f]_{\boldsymbol{v}}(t, \boldsymbol{x})$ with use of the linearized collision operator given by

$$
\left.L_{\boldsymbol{v} \boldsymbol{k}} \equiv\left(f_{\boldsymbol{v}}^{\mathrm{eq}}\right)^{-1} \frac{\partial}{\partial f_{\boldsymbol{k}}} C[f]_{\boldsymbol{v}}\right|_{f=f^{\mathrm{eq}}} f_{\boldsymbol{k}}^{\mathrm{eq}} .
$$

Thus, the Grad-Müller equation is obtained as a closed system of the equations governing $T, n, u^{i}, \pi^{i j}$, and $J^{i}$ in terms of the transport coefficients and the relaxation times, which are given in terms of an inner product defined by $\langle\psi, \chi\rangle_{\text {eq }} \equiv \sum_{\boldsymbol{v}} f_{\boldsymbol{v}}^{\text {eq }} \psi_{\boldsymbol{v}} \chi_{\boldsymbol{v}}$. For example, the shear viscosity and the relaxation time of the stress tensor $\hat{\pi}^{i j}$ are expressed as

$$
\begin{aligned}
\eta^{\mathrm{G}} & =-\frac{1}{10 T} \frac{\left\langle\hat{\pi}^{i j}, \hat{\pi}^{i j}\right\rangle_{\mathrm{eq}}\left\langle\hat{\pi}^{k l}, \hat{\pi}^{k l}\right\rangle_{\mathrm{eq}}}{\left\langle\hat{\pi}^{m n}, L \hat{\pi}^{m n}\right\rangle_{\mathrm{eq}}}, \\
\tau_{\pi}^{\mathrm{G}} & =-\frac{\left\langle\hat{\pi}^{i j}, \hat{\pi}^{i j}\right\rangle_{\mathrm{eq}}}{\left\langle\hat{\pi}^{k l}, L \hat{\pi}^{k l}\right\rangle_{\mathrm{eq}}} .
\end{aligned}
$$

It is well known that the formula (6.25) is different from that given in the Chapman-Enskog expansion method, and there are many attempts both in non-relativistic [44$47]$ and relativistic cases [24,57] to modify and/or extend the Grad moment method so that the transport coefficients thus obtained become consistent with those obtained by Chapman-Enskog method.

\subsection{Relativistic mesoscopic dynamics from the RG method}

In this subsection, we make a brief report on our attempt [40] to extend the RG method so as to obtain the so called mesoscopic dynamics $[42,43]$ in the relativistic case.

First we show the results in such a way that a comparison with the Grad moment method is apparent. If we express the distribution function by $f_{p}(x)=f_{p}^{\text {eq }}(x)(1+$ $\Phi_{p}(x)$ ), our RG method gives the following expression of $\Phi_{p}(x)$,

$$
\Phi_{p}=-\frac{1}{T} \sum_{q} L_{p q}^{-1}\left(\tilde{\Pi}_{q} \frac{\Pi}{\zeta}+\tilde{J}_{q}^{\mu} \frac{J_{\mu}}{\lambda}+\tilde{\pi}_{q}^{\mu \nu} \frac{\pi_{\mu \nu}}{2 \eta}\right)
$$

where $\tilde{\Pi}_{p}, \tilde{J}_{p}^{\mu}$, and $\tilde{\pi}_{p}^{\mu \nu}$ are the microscopic thermal forces. This new form is different from any proposals in the literature $[24,57]$.

The resultant energy-momentum tensor and particle current are found [40] to have the following forms, respectively:

$$
\begin{aligned}
T_{2 \mathrm{nd}}^{\mu \nu} & =e u^{\mu} u^{\nu}-(p+\Pi) \Delta^{\mu \nu}+\pi^{\mu \nu}, \\
N_{2 \mathrm{nd}}^{\mu} & =n u^{\mu}+J^{\mu} .
\end{aligned}
$$

The relaxation equations derived in our RG method read

$$
\begin{aligned}
\Pi= & -\zeta \nabla \cdot u-\tau_{\Pi} D \Pi \\
& + \text { other terms involving relaxation lengths, (6.30) } \\
J^{\mu}= & \lambda \frac{1}{\hat{h}^{2}} \nabla^{\mu} \frac{\mu}{T}-\tau_{J} \Delta^{\mu a} D J_{a} \\
& + \text { other terms involving relaxation lengths, (6.31) } \\
\pi^{\mu \nu}= & 2 \eta \Delta^{\mu \nu \rho \sigma} \nabla_{\rho} u_{\sigma}-\tau_{\pi} \Delta^{\mu \nu a b} D \pi_{a b} \\
& +\left(\kappa_{\pi \pi}^{(0)} \Delta^{\mu \nu \rho \sigma} \nabla \cdot u+\kappa_{\pi \pi}^{(1)} \Delta^{\mu \nu a c} \Delta_{c}^{b \rho \sigma} \Delta_{a b d e} \nabla^{d} u^{e}\right. \\
& \left.+\kappa_{\pi \pi}^{(2)} \Delta^{\mu \nu a c} \Delta_{c}^{b \rho \sigma} \omega_{a b}\right) \pi_{\rho \sigma} \\
& + \text { other terms involving relaxation lengths, }
\end{aligned}
$$

where $\omega^{\mu \nu} \equiv \frac{1}{2}\left(\nabla^{\mu} u^{\nu}-\nabla^{\nu} u^{\mu}\right)$ is the vorticity.

Our RG method [40] gives microscopic expressions for the relaxation times $\tau_{\Pi}, \tau_{J}$, and $\tau_{\pi}$ as follows:

$$
\tau_{\Pi} \equiv-\frac{\left\langle\tilde{\Pi}, L^{-2} \tilde{\Pi}\right\rangle}{\left\langle\tilde{\Pi}, L^{-1} \tilde{\Pi}\right\rangle}=\frac{\int_{0}^{\infty} \mathrm{d} s s R_{\zeta}(s)}{\int_{0}^{\infty} \mathrm{d} s R_{\zeta}(s)},
$$

and so on for $\tau_{J}$ and $\tau_{\pi}$ with obvious modifications. We note that our novel formulae for the relaxation times are all nicely represented in terms of the relaxation functions 
$R_{\zeta}(s), R_{\lambda}(s)$, and $R_{\eta}(s)$ so that they have a natural physical meaning of the relaxation time as the correlated time of the respective relaxation function in contrast to other approaches $[24,49,57]$. We also mention that bulk and shear viscosities and the heat conductivity derived in our method do coincide with those in the Chapman-Enskog method as shown before.

\section{Summary and concluding remarks}

We have reported our attempts to derive first-order and second-order relativistic hydrodynamic equations from relativistic Boltzmann equation which has a manifest Lorentz invariance and does not show any pathological behavior such as the instability and acausality seen in existing hydrodynamic equations. We have given an argument, on a physical ground on the nature of the origin of the dissipation and the form of the local equilibrium distribution function, that the energy frame is uniquely chosen as the one in which the relativistic hydrodynamic equation for a viscous fluid is defined. We have given the novel extended thermodynamics both in non-relativistic and relativistic cases through the explicit construction of attractive manifold containing the relaxation process from Boltzmann equation.

It is worth emphasizing that all the equations derived in this work are consistent with the underlying kinetic equation, i.e., relativistic Boltzmann equation. This is one of the advantage in our theory because our theory explicitly gives the solution (distribution function) of the Boltzmann equation which is expressed with the hydrodynamic variable and relaxation times and lengths, and thereby makes a systematic description of the time-evolution of the system from hydrodynamic to kinetic regime. Such an overall analysis should be desirable for that of the matter created at RHIC, LHC and other systems where the proper dynamics would change from the hydrodynamic to the kinetic ones or vice versa [4-9]. Furthermore, it would be interesting to evaluate the relaxation times as well as the transport coefficients of the created matter with the use of the microscopic representations obtained in this work.

Finally, we note that the renormalization-group method $[32-37,51,52]$ itself has a universal nature and can be applied to derive a slow dynamics from kinetic equations other than the simple Boltzmann equation, say, KadanoffBaym equation [58].

TK thanks the editors to invite him to contribute to this special issue in The European Physical Journal A. We are grateful to K. Ohnishi for his collaboration in the early stage of this work. TK was partially supported by a Grant-in-Aid for Scientific Research from the Ministry of Education, Culture, Sports, Science and Technology (MEXT) of Japan (Nos. 20540265 and 23340067), by the Yukawa International Program for QuarkHadron Sciences, and by a Grant-in-Aid for the global COE program "The Next Generation of Physics, Spun from Universality and Emergence" from MEXT.
Open Access This is an open access article distributed under the terms of the Creative Commons Attribution License (http://creativecommons.org/licenses/by/3.0), which permits unrestricted use, distribution, and reproduction in any medium, provided the original work is properly cited.

\section{References}

1. P. Huovinen, in Quark Gluon Plasma 3, edited by R.C. Hwa, X.N. Wang (World Scientific, Singapore, 2003) p. 600 and P.F. Kolb, U.W. Heinz, in Quark Gluon Plasma 3, edited by R.C. Hwa, X.N. Wang (World Scientific, Singapore, 2003) p. 634.

2. M. Gyulassy, L. McLerran, Nucl. Phys. A 750, 30 (2005).

3. P. Bozek, Acta Phys. Pol. B 43, 689 (2012).

4. T. Hirano, P. Huovinen, K. Murase, Y. Nara, to be published in Prog. Part. Nucl. Phys., arXiv:1204.5814 [nuclth].

5. S.A. Bass, A. Dumitru, Phys. Rev. C 61, 064909 (2000).

6. D. Teaney, J. Lauret, E.V. Shuryak, Phys. Rev. Lett. 86, 4783 (2001).

7. T. Hirano, M. Gyulassy, Nucl. Phys. A 769, 71 (2006).

8. T. Hirano, U.W. Heinz, D. Kharzeev, P. Lacey, Y. Nara, Phys. Lett. B 636, 299 (2006).

9. C. Nonaka, S.A. Bass, Phys. Rev. C 75, 014902 (2007).

10. H. Fujii, M. Ohtani, Phys. Rev. D 70, 014016 (2004).

11. D.T. Son, M.A. Stephanov, Phys. Rev. D 70, 056001 (2004).

12. Y. Minami, T. Kunihiro, Prog. Theor. Phys. 122, 881 (2010).

13. M. Asakawa, K. Yazaki, Nucl. Phys. A 504, 668 (1989).

14. A. Barducci, R. Casalbuoni, S. De Curtis, R. Gatto, G. Pettini, Phys. Lett. B 231, 463 (1989).

15. Z. Zhang, T. Kunihiro, Phys. Rev. D 83, 114003 (2011).

16. C. Eckart, Phys. Rev. 58, 919 (1940).

17. L.D. Landau, E.M. Lifshitz, Fluid Mechanics (Pergamon Press, London, 1959).

18. P. Van, T.S. Biro, Phys. Lett. B 709, 106 (2012).

19. T. Osada, Phys. Rev. C 85, 014906 (2012).

20. W.A. Hiscock, L. Lindblom, Phys. Rev. D 31, 725 (1985).

21. S.R. de Groot, W.A. van Leeuwen, Ch.G. van Weert, Relativistic Kinetic Theory (Elsevier, North-Holland, 1980).

22. C. Cercignani, G.M. Kremer, The Relativistic Boltzmann Equation (Birkhauser, Berlin, 2002).

23. W. Israel, Ann. Phys. 100, 310 (1976).

24. W. Israel, J.M. Stewart, Ann. Phys. 118, 341 (1979).

25. K. Tsumura, T. Kunihiro, K. Ohnishi, Phys. Lett. B 646 , 134 (2007).

26. K. Tsumura, T. Kunihiro, Phys. Lett. B 690, 255 (2010).

27. K. Tsumura, T. Kunihiro, Prog. Theor. Phys. 126, 761 (2011).

28. N.N. Bogoliubov, in Studies in Statistical Mechanics, edited by J. de Boer, G.E. Uhlenbeck, Vol. 1 (NorthHolland, 1960).

29. R. Kubo, M. Toda, N. Hashitsume, Statistical Physics II, in Springer Series in Solid-State Sciences, Vol. 31 (Springer, Berlin, 1991) and D. Zubarev, V. Morozov, G. Roepke, Statistical Mechanics of Nonequilibrium Processes, Vols. 1 and 2 (Akademie Verlag GmbH, Berlin, 1996, 1997) and L.E. Reichl, A Modern Course in Statistical Physics, 2nd edition (John Wiley and Sons, New York, 1998).

30. M. Dudynski, M.L. Ekiel-Jezewska, Phys. Rev. Lett. 55, 2831 (1985). 
31. R.M. Strain, Commun. Math. Phys. 300, 529 (2010).

32. L.Y. Chen, N. Goldenfeld, Y. Oono, Phys. Rev. Lett. 73, 1311 (1994).

33. L.Y. Chen, N. Goldenfeld, Y. Oono, Phys. Rev. E 54, 376 (1996).

34. T. Kunihiro, Prog. Theor. Phys. 94, 503 (1995) 95, 835(E) (1996)

35. T. Kunihiro, Jpn. J. Ind. Appl. Math. 14, 51 (1997).

36. T. Kunihiro, Prog. Theor. Phys. 97, 179 (1997).

37. S.-I. Ei, K. Fujii, T. Kunihiro, Ann. Phys. 280, 236 (2000).

38. J. Guckenheimer, P. Holmes, Nonlinear Oscillators, Dynamical Systems, and Bifurcations of Vector Fields (Springer-Verlag, 1983).

39. Y. Kuramoto, Prog. Theor. Phys. Suppl. 99, 244 (1989).

40. K. Tsumura, T. Kunihiro, in preparation (a preliminary report is given in [53]).

41. I. Müller, T. Ruggeri, Extended Thermodynamics (Springer-Verlag, Berlin, 1993).

42. D. Jou, J. Casas-Vazquez, G. Lebon, Extended Irreversible Thermodynamics (Springer-Verlag, 1993).

43. T. Dedeurwaerdere, J. Casas-Vazquez, D. Jou, G. Lebon, Phys. Rev. E 53, 498 (1996).
44. Iliya V. Karlin, Alexander N. Gorban, G. Dukek, T.F. Nonnenmacher, Phys. Rev. E 57, 1668 (1998).

45. P.A. Zakharchenko, E.V. Radkevich, Doklady Phys. 49, 451 (2004).

46. H.C. Öttinger, Phys. Rev. Lett. 104, 120601 (2010).

47. M. Torrilhon, Commun. Comput. Phys. 7, 639 (2010).

48. S. Chapman, T.G. Cowling, The Mathematical Theory of Non-Uniform Gases (Cambridge University Press, UK, 1939).

49. H. Grad, Comm. Pure Appl. Math. 2, 331 (1949).

50. F. Jüttner, Ann. Phys. Chem. 339, 856 (1911).

51. Y. Hatta, T. Kunihiro, Ann. Phys. 298, 24 (2002).

52. T. Kunihiro, K. Tsumura, J. Phys. A 39, 8089 (2006).

53. K. Tsumura, T. Kunihiro, Prog. Theor. Phys. Suppl. 195, 19 (2012) arXiv:1205.5843 [nucl-th].

54. K. Tsumura, T. Kunihiro, arXiv:1206.3913.

55. Y. Minami, Y. Hidaka, private communication.

56. K. Tsumura, T. Kunihiro, Phys. Lett. B 668, 425 (2008).

57. G.S. Denicol, T. Koide, D.H. Rischke, Phys. Rev. Lett. 105, 162501 (2010).

58. L.P. Kadanoff, G. Baym, Quantum Statistical Mechanics (W.A. Benjamin, N.Y., 1962). 